\title{
Anton Howes, Arts and Minds: How the Royal Society of Arts Changed a Nation
}

New Jersey, Princeton University Press, 2020

\section{Yohann Guffroy}

\section{(2) OpenEdition}

\section{Journals}

Édition électronique

URL : http://journals.openedition.org/artefact/7133

DOI : 10.4000/artefact.7133

ISSN : 2606-9245

\section{Éditeur :}

Association Artefact. Techniques histoire et sciences humaines, Presses universitaires du Midi

\section{Édition imprimée}

Date de publication : 7 janvier 2021

Pagination : 465-470

ISBN : 978-2-8107-0706-5

ISSN : 2273-0753

Référence électronique

Yohann Guffroy, «Anton Howes, Arts and Minds: How the Royal Society of Arts Changed a Nation »,

Artefact [En ligne], 13 | 2020, mis en ligne le 23 décembre 2020, consulté le 27 décembre 2020. URL: http://journals.openedition.org/artefact/7133 ; DOI : https://doi.org/10.4000/artefact.7133

Ce document a été généré automatiquement le 27 décembre 2020.

\section{c) (i) $९$}

Artefact, Techniques, histoire et sciences humaines est mise à disposition selon les termes de la Licence Creative Commons Attribution - Pas d'Utilisation Commerciale - Pas de Modification 4.0 International. 


\section{Anton Howes, Arts and Minds: How the Royal Society of Arts Changed a Nation}

New Jersey, Princeton University Press, 2020

\section{Yohann Guffroy}

\section{RÉFÉRENCE}

Anton Howes, Arts and Minds: How the Royal Society of Arts Changed a Nation, New Jersey, Princeton University Press, 2020, 387 p.

1 L'ouvrage d'Anton Howes, Arts and Minds, est une nouvelle histoire générale de la Royal Society for the Encouragement of Arts, Manufacture and Commerce (RSA) rédigée près de soixante-dix ans après la dernière ${ }^{1}$. Le livre est d'un intérêt indéniable au regard de ses prédécesseurs ${ }^{2}$. En plus d'aborder la seconde moitié du $\mathrm{xx}^{\mathrm{e}}$ siècle, Howes propose ici une histoire « hors les murs » de l'institution, une histoire qui ne s'attache pas tant à décrire son évolution interne qu'à étudier la manière dont la RSA se positionne à travers le temps au sein de la société anglaise. Cette approche est nécessairement à double tranchant puisque ceux qui souhaiteraient connaître les détails du fonctionnement organique de la Société devront se reporter aux deux précédentes histoires ; cependant, grâce à cette perspective, Howes parvient à mettre précisément en évidence l'inscription de la RSA dans le tissu social comme lieu à la fois de promotion d'une idée du bien public (public good), de gestation de réformes et de débats.

2 L'ouvrage est illustré de plusieurs planches et se compose de treize chapitres qui peuvent être regroupés en trois temps de vie de l'institution. Le premier s'étend de 1754 à 1851 (chapitres I à IV), le deuxième de 1851 au début des années 1900 (chapitres $\mathrm{V}$ à IX) et le troisième du début du XX $\mathrm{XX}^{\mathrm{e}}$ siècle à nos jours (chapitres $\mathrm{X}$ à XIII). Howes rappelle dès les premières pages que le mouvement des sociétés savantes en 
Angleterre et en Europe aux $\mathrm{XVII}^{\mathrm{e}}$ et $\mathrm{XVIII}^{\mathrm{e}}$ siècles s'inscrit plus largement dans un " programme baconien ${ }^{3}$ » de collecte et de partage de connaissances (p. 1). La RSA, fondée en 1754 par l'artiste peintre William Shipley, n'échappe pas à la règle. Son fondateur est, selon l'auteur, un produit de cette tradition qui sous-tend l'idéal du projet (p. 10). La Société naît autour d'une table d'un café de Londres de l'association d'un groupe d'hommes mené par Shipley qui ont pour but de promouvoir une idée de bien commun et de progrès au sein de la société anglaise.

Durant son premier siècle d'existence, la Société s'emploie à promouvoir les «beauxarts » (polite arts), tels que le dessin, la peinture ou la sculpture, et l'industrie par l'intermédiaire de prix décernés à des candidats répondant aux mises au concours ${ }^{4}$. Les productions sont évaluées par des comités organisés selon différents domaines (agriculture, chimie, mécanique, manufacture, polite arts, colonies et commerce). Howes analyse les premiers temps de l'action de la RSA à travers le prisme de la compétition entre la France et la Grande-Bretagne. Il s'agit à la fois de concurrencer l'art français mais aussi de stimuler l'industrie anglaise par la production d'inventions, qu'il s'agisse de machines ou de procédés en agriculture, marine, textile, etc. D'autre part, la RSA cherche à améliorer les conditions de vie de la population et s'occupe de sujets aussi divers que le travail des enfants ramoneurs, la prostitution ou encore la lutte contre la falsification des billets de banque particulièrement lors de la crise bancaire de la fin des années 1790.

4 À partir des années 1820-1830, le caractère technique de la Société se renforce peu à peu du fait de l'arrivée de nouveaux membres, en particulier les ingénieurs, les manufacturiers et les agents de brevet. Ces nouvelles catégories entraînent de fait une spécialisation et une professionnalisation des comités d'examen. Dans le même temps, la Société fait face à une crise financière et frôle la faillite dans les années 1840. Cet événement oblige son secrétaire de l'époque, Francis Whishaw, à repenser le modèle de fonctionnement de l'institution. Son idée est alors de développer les expositions techniques sur le modèle de ce que propose la France dans les mêmes années. La thématique de l'exposition n'est pas étrangère à la RSA puisqu'elle en a organisé plusieurs durant la seconde moitié du XviII ${ }^{\mathrm{e}}$ siècle, qu'elles aient été artistiques ou techniques. Whishaw projette même plus grand, une exposition industrielle d'envergure nationale qu'il ne mènera cependant pas à bien. Le projet sera repris et amplifié par un membre ambitieux de la Société, Henry Cole, promoteur de l'Exposition universelle de 1851.

5 À la différence d'Hudson et Luckhurst qui considéraient le temps de l'Exposition comme l'événement de rupture entre la première et la deuxième phase de vie de la Société, Howes préfère envisager cette dernière du point de vue de la figure de Cole. En effet, si jusqu'ici la Société était présentée comme une entité qui prenait des décisions collégiales, les chapitres qui lui sont consacrés montrent comment l'institution devient entre ses mains un outil lui permettant de promouvoir ses propres idées utilitaristes. Son objectif est simple : éduquer la population, notamment les travailleurs, et la mettre en contact avec le «beau». L'orientation vers le modèle des expositions provoque cependant des tensions au sein de la RSA, les ingénieurs déplorant l'abandon progressif de l'incitation à l'invention (p.138-139). Afin de faire taire les contestations, Cole démissionne du conseil de la Société pour permettre la tenue de nouvelles élections qu'il remporte. Cette victoire ouvre une nouvelle phase dans l'histoire de l'institution. 
6 La Société est vue par Cole comme un moyen de pousser un certain nombre de réformes. Il participe à celle des brevets d'invention et est l'initiateur de celle du copyright des productions artistiques (peintures, dessins, photographies) qui sera adoptée en 1862 par le Parlement. Il tente également d'investir le domaine de la santé publique lors de l'Exposition en cherchant à installer un système de toilettes publiques. L'essai est toutefois un échec et laisse croire au gouvernement que la demande n'existe pas ce qui retarde de plusieurs années leur déploiement (p. 149-151). Cole n'en oublie pas moins sa volonté de développer une éducation à l'esthétique et au design. Il se voit d'abord nommer superintendant du Department of Practical Arts qui lui permet de contrôler à la fois l'École de Design de Londres et un musée établi du côté de St James's Park à partir des modèles de cette dernière. En 1857, Cole déplace ce musée dédié aux productions manufacturières dans le district de Brompton. Il le renomme alors South Kensington Museum qui deviendra plus tard le Victoria \& Albert Museum.

7 Du point de vue de l'engagement de l'institution dans l'éducation populaire, différentes initiatives sont soutenues : l'Union des Instituts mécaniques fondée en 1852 ; la mise en place progressive d'un système d'examen organisé et géré par un Conseil des examens financé par le gouvernement permettant de sanctionner les compétences acquises par les travailleurs puis par les jeunes sortant des écoles; le lancement d'un programme d'éducation alimentaire (Food of the People) à destination des familles les plus pauvres avec notamment une exposition; l'initiative de Maria Grey pour la mise en place d'écoles pour jeunes filles et plus généralement la mise à l'école de tous les jeunes enfants qui se traduit par la loi sur l'école élémentaire de 1870 (p. 192). Ces différents engagements occupent la Société toute la fin du xix siècle et une partie des années 1900 à l'exception de sa participation au Conseil des examens qui se poursuit jusque dans les années 1980.

8 La troisième partie de l'ouvrage couvre le $\mathrm{xx}^{\mathrm{e}}$ siècle. Cette période est pour la RSA un temps d'incertitude, de remise en question et de quête d'une nouvelle vision d'ensemble. Durant la première moitié du siècle, la Société s'en tient à son engagement dans le Conseil des examens et quelques actions dans la protection des campagnes anglaises et des bâtiments dits « historiques ». C'est aussi le temps de l'introduction en son sein d'une classe «managériale » constituée d'employés d'entreprises, d'industriels et même d'employés des ministères. Leur intérêt pour la RSA est motivé par la possibilité de partager à la fois leur activité mais aussi leurs goûts pour les arts et la science. L'un des exemples cités est celui d'Arthur Roberts, représentant de la firme Plant Protection Ltd. spécialisée dans les pesticides, qui convainc en 1945 sa société de donner une série de conférences sur l'agronomie et particulièrement sur les effets des herbicides (p. 249).

9 Bien que la Société accueille ce type d'interventions, cela ne l'empêche pas à partir des années 1960 de lancer, par l'intermédiaire de son Président le Prince Philip, une réflexion sur la protection de l'environnement. On notera que ce passage du livre s'appuie sur un entretien de l'auteur avec l'intéressé. Howes rapporte que c'est après la lecture du livre Silence Spring ${ }^{5}$ de Rachel Carson publié en 1962 que le Prince Philip s'engage pleinement dans la réflexion écologique en promouvant publiquement l'ouvrage, en l'envoyant à tous les ministres du gouvernement d'alors et en instaurant en 1971 un comité environnemental au sein de la RSA. Ce dernier met par exemple en place un prix pour inciter les entreprises à trouver des solutions aux dégâts causés par leur activité (p. 262). 
10 Malgré ces quelques éléments, la période 1910-1980 signe une absence de vision à long terme de la Société, du moins si l'on en croit l'auteur. Il revient à son directeur du moment, Christopher Taylor d'amorcer à partir de 1977 un renouveau au sein de l'institution. L'année 1987 est notamment présentée comme charnière. En effet, Taylor décide à cette date de quitter le Conseil des examens et par la même occasion de se couper de ses sources de financement gouvernementales. Cette décision est lourde de conséquences puisque le quitter revient à mettre en danger le budget de la RSA. Taylor recentre alors l'activité de la Société sur les axes déjà existants: l'environnement, l'éducation et le design en maintenant le prix du Royal Designers for Industry créé en 1938. Ce recentrage permet l'introduction de nouvelles figures au sein de l'institution et notamment des femmes dont Howes cite deux exemples: Prue Leith et Penny Egan. La première mène avec la Société un projet d'éducation alimentaire appelé Focus on Food qui a pour but de mener des interventions dans les écoles pour initier les enfants à une culture de l'alimentation (p. 290-295). La seconde devient en 1998 la première directrice de la RSA et promotrice, en accord avec son parcours, de l'enseignement de l'art à l'école (p. 296-302). On remarquera que l'introduction de ces figures importantes à la fois dans l'histoire de l'institution et de la société anglaise sert de marqueur de rupture pour Howes qui relève qu'au cours de la direction d'Egan, le nombre de femmes au sein de la RSA passe de 5 à $30 \%$ en dix ans.

11 L'auteur ne conclut pas réellement son ouvrage mais propose un dernier chapitre sous forme de question: Building a Social Movement? Dans les quelques dernières pages, il retrace à grands traits la manière dont la Société a essayé au fil de son histoire de fédérer un large public autour de ses projets, ses réflexions et ses actions. C'est d'abord une tentative d'expansion qui est amorcée avec la création de comités de la Société des arts dans les campagnes, l'intégration de membres venus des colonies et du Commonwealth, ou encore l'ouverture à différentes catégories sociales. Mais il s'est aussi agi pour la Société de faire son introspection et de se réformer afin de redéfinir une trajectoire claire pour porter au mieux les différents domaines dans lesquelles elle est engagée.

12 Tout l'intérêt du livre d'Anton Howes est de nous permettre d'approcher divers aspects de l'histoire anglaise de ces deux cent cinquante dernières années par l'intermédiaire de celle d'une société savante engagée au service de son pays. On relèvera toutefois une ambiguïté à la lecture de certains passages de la première partie où le mot " patriotic » est assez fréquemment utilisé sans que l'on sache clairement s'il s'agit d'une qualification d'époque ou de l'auteur. Par ailleurs, ce dernier laisse des zones d'ombre que ni lui ni ses prédécesseurs n'ont éclairé. Telle qu'elle est présentée ici, l'histoire de la RSA est jusque dans les années 1980 une histoire d'hommes et les femmes semblent y avoir très peu leur place. Bien qu'elles aient été autorisées dès l'origine à en devenir membre, Howes indique qu'il est difficile de savoir quelle part elles ont pu prendre dans son fonctionnement. On renverra cependant le lecteur sur ce point aux travaux de Marina Giardinetti qui s'emploie à étudier cet aspect ${ }^{6}$. On ajoutera enfin que les planches jointes au texte principal ne sont pas du tout exploitées et ne servent que d'illustrations pour donner à voir quelques productions des membres de la RSA. Toute analyse de ces images et de leurs auteurs est négligée, délaissant de fait tout un pan de l'activité de la Société. Les travaux de Marina Giardinetti' et notre propre travail de thèse ${ }^{8}$ cherchent cependant à pallier ce manque. 


\section{BIBLIOGRAPHIE}

CARSON Rachel, Silence Spring, Houghton Mifflin Company, Boston, 1962.

GIARDINETTI Marina, «Exercices de style : éducation et pratiques artistiques des jeunes femmes de la Society of Arts ", mémoire de master sous la direction de Liliane Hilaire-Pérez, Université de Paris, 2019.

GIARDINETTI Marina, « Être dessinateur ou dessinatrice à la Society of Arts de Londres au XVIII ${ }^{\mathrm{e}}$ siècle : quels apprentissages ? ", Artefact. Techniques, histoire et sciences humaines (à paraître). HILAIRE-PÉREZ Liliane, L'Invention technique au siècle des Lumières, Albin Michel, Paris, 2000.

HUDSON Derek, LUCKHURST Kenneth W., History of the Royal Society of Arts, 1754-1954, John Murray, Londres, 1954.

MOKYR Joel, « The Intellectual Origins of Modern Economic Growth », The Journal of Economic History, 65, n² 2, juin 2005, p. 258-351.

TRUEMAN WOOD Henry, The History of the Royal Society of Arts, John Murray, Londres, 1913.

\section{NOTES}

1. Hudson et Luckhurst, 1954.

2. Une première histoire générale est publiée en 1913 par le Secrétaire d'alors, Henry Trueman Wood.

3. L'auteur reprend l'expression proposée par l'historien Joel Mokyr. Mokyr, 2005, p. 289.

4. Pour une analyse des prix proposés par la Society of Arts du XVIII ${ }^{\mathrm{e}}$ siècle, voir Hilaire-Pérez, 2000.

5. Carson, 1962.

6. Voir par exemple Giardinetti, 2019.

7. Giardinetti, 2021.

8. Notre recherche de thèse menée à l'École polytechnique fédérale de Lausanne sous la direction de Jérôme Baudry et Liliane Hilaire-Pérez porte sur l'évolution du dessin d'objet technique en Angleterre entre 1750 et 1850 . Les principaux corpus mobilisés sont ceux de la Royal Society of Arts, des specifications des patents et du commerce par l'entremise des trade cards et des trade books.

\section{AUTEURS}

\section{YOHANN GUFFROY}

Laboratoire d'histoire des sciences et des techniques-EPFL 